\title{
Changes of retinal thickness in macular area of myopia children
}

\author{
Zhu Meihong ${ }^{1 *}$ Lin Tainan $^{2}$ \\ ${ }^{1}$ Huaqiao University Hospital, Ophthalmology, Quanzhou City, Fujian Province , 362021, China \\ ${ }^{2}$ Fujian Provincial government Hospital, Ophthalmology, Quanzhou City, Fujian Province, 350003, China
}

\begin{abstract}
Objective: To study the changes of macular retinal thickness in children with moderate and low myopia by frequency domain OCT. Methods: 108 school-age children aged 7-12 years were selected and enrolled into the group. Among them, 58 cases (116 eyes) with simple moderate and low myopia in the myopia group and 50 cases (100 eyes) with straight eyes in the emmetropia group. The retinal thickness in different parts of macular area was compared between the two groups, and the correlation between retinal thickness in different parts of macular area and diopter and axial length was analyzed by Pearson correlation analysis. Results: The length of eye axis in moderate and low myopia group was longer than that in emmetropia group, and the difference was statistically significant $(\mathrm{P}<0.05)$. There was no significant difference in CMT(Central Macular Thickness) and I-AMT(average macular thickness of inner circle) between the two groups $(\mathrm{P}>0.05)$. The AMT(Average Macular Thickness) and O-AMT(average macular thickness of outer circle) in moderate and low myopia group were lower than those in emmetropia group, and the differences were all statistically significant $(\mathrm{P}<0.05)$. The thickness of the retina below the macula and the nasal side was positively correlated with the refractive power in patients with moderate to low-grade myopia ( $\mathrm{r}=0.201$, $0.302, \mathrm{P}<0.05)$, and the thickness of the retina around the macula was negatively correlated with the refractive power $(\mathrm{r}=-0.326, \mathrm{P}<0.05)$. Conclusion: There is no significant difference in macular central retinal thickness between children with moderate and low myopia and normal children, and the thickness of paracentral retina tends to become thinner with the deepening of myopia.
\end{abstract}

\section{Introduction}

Refraction is a common eye disease in children, which is the main cause of poor eyesight in children. With the development of society and the progress of science and technology, the incidence of ametropia in children is increasing year by year. Compared with emmetropia, myopia has many degrees of retinal changes [1], including thinning of nerve fiber layer, leopard-print fundus, lacquered crack, myopic atrophy arc, neovascularization, etc. Such fundus changes are more likely to cause corresponding complications, which is the main pathological change of blindness in myopia patients. When the thickness of retinal nerve fiber layer becomes thinner, the retinal nerve fiber layer will shrink, which will eventually cause the atrophy of optic nerve, resulting in the reduction of visual field and the decline of visual function [23]. Therefore, it is of positive significance to evaluate the thickness of retinal nerve fiber layer for evaluating fundus diseases of myopia patients.

OCT can accurately obtain RNFLT (Retinal Fiber Layer Thickness) many times without touching human body. Its imaging principle is that when scanning tissues at different depths, the weak coherent light received at the biological tissue level will be reflected and scattered backward, thus forming the corresponding OCT image, which has light and dark gray scale changes. Optical coherence tomography mainly includes two types: time domain and frequency domain. OCT in frequency domain combines spectral analysis with optical coherence imaging technology to collect information of different wavelengths, which is presented in the form of depth information after computer analysis [3]. OCT has two resolutions, which have different positions in the scanning process of OCT, and the axial resolution is particularly important. In addition to axial resolution, OCT also has an indispensable lateral resolution, which is determined by the spot illuminating the object to be measured. Therefore, we can use the focused light source to illuminate the object, so as to reduce the light spot and improve the lateral resolution.

Study [4] found that the lengthening of eye axis increased the risk of pathological changes of retina. This study measured the length of eye axis and retinal thickness of macular area in 58 middle and low myopia and 50 low myopia, and analyzed the related factors of macular thickness change, which can promote the correct evaluation of myopia and the prevention of complications of ultra-high school-age children. 


\section{Measurement of RNFLT in myopia patients by OCT}

The number of myopia has reached 500 million in China, and it is on the rise year by year. With the increase of the number of myopia patients, people pay more and more attention to the changes of fundus. Compared with emmetropia, myopia has many degrees of retinal changes [5], including thinning of nerve fiber layer, leopard-print fundus, lacquered crack, myopic atrophy arc, neovascularization, etc. Such fundus changes are more likely to cause corresponding complications, and are the main pathological changes causing blindness in myopia patients. Among these retinopathy, the change of retinal nerve fiber layer thickness is one of the most influential retinopathy. Cirrus HD-OCT was used to analyze 98 eyes with different diopters, and it was found that the average value of RNFLT around the optic papilla of moderate and high myopia patients was smaller than that of low myopia patients. Literature [6] OCT was used to measure RNFLT around optic papilla in 48 myopia patients. The study showed that the average RNFLT of high myopia patients was obviously smaller than that of low myopia.

Literature [7] 15 children with high myopia and 20 children with mild myopia aged from 4 to 10 years were studied by OCT. It was found that the average RNFLT around the optic papillae in high myopia group was 100.8 um, while the average RNFLT around the optic papillae in light myopia group was $110.5 \mathrm{um}$. The average RNFLT around the optic papillae in high myopia group was significantly thinner than that in light myopia group, and the RNFLT under the optic papillae became thinner in high myopia group. Literature [8] The RNFLT around the optic papilla was measured by OCT in 120 eyes of 120 children. It was found that the RNFLT around the optic papilla in myopia group was thinner than that in hyperopia group and emmetropia group in 40 eyes. [9] After grouping 135 children (270 eyes) aged 10-12 years, it was found that the RNFLT on the nasal side of the optic papilla in high myopia group was thinner than that in moderate myopia group, mild myopia group and positive control group.

Literature [10] 115 healthy control eyes, 75 high myopia eyes and 40 low-moderate myopia eyes were selected, and the RNFLT around the optic papilla was measured by OCT. It was found that the retinal nerve fiber layer on the nasal side of myopia subjects was thin. Literature [11] After using OCT to measure RNFLT around the optic papilla in 106 cases (196 eyes) of myopia and 38 cases (60 eyes) of normal control group, it was found that the average thickness of RNFLT around the optic papilla and the upper and lower quadrant RNFLT in each myopia group were thinner than those in normal control group.

\section{$3 \quad$ Materials and methods}

\subsection{General information}

School-age children aged 7 12 years in our hospital were selected continuously, except retinal diseases, optic nerve diseases or glaucoma family history, ocular trauma history, and systemic diseases that may affect the eyes. There was no abnormality in the anterior segment and fundus examination, the best corrected distance vision was $\geq 1.0$, and the equivalent spherical power (SE) was-6.00 D $+0.50 \mathrm{D}$.

A total of 108 cases (216 eyes) were enrolled, with an average age of $(9.39 \pm 1.41)$ years. Among them, 58 cases (116 eyes) with simple moderate and low myopia in both eyes were enrolled in the myopia group, with an average age of $(9.30 \pm 1.34)$ years, and the equivalent spherical degree was $(-2.50 \pm 1.33) \mathrm{D}$, with an average of $(-2.50$ D), including 30 males and 28 females.

In the emmetropia group, 50 children (100 eyes) with binocular vision, with an average age of $(9.68 \pm 1.53)$ years, an equivalent spherical power of $-0.50 \mathrm{D} \sim+0.50$ $\mathrm{D}$, and an average of $(+0.25 \pm 0.23) \mathrm{D}$, including 28 males and 22 females.

\subsection{Inspection method}

\section{(1) Measurement of diopter}

Ciliary body paralysis agent (1\% Atropine eye ointment) was used to spot both eyes twice a day a day for three days. After three days, the pupil's light reflection was observed. After the pupil's light reflection disappeared, computer optometry was performed (automatic computer optometry instrument Topcon RM-8800), and the diopter was calculated according to the equivalent spherical mirror (equivalent spherical mirror $=$ spherical mirror $+1 / 2$ cylindrical mirror).

(2) OCT examination

The thickness of retina in different quadrants of macular area was measured in middle and low myopia group and emmetropia group. During the examination, the patient was seated, the lower jaw was placed on the jaw frame, and the method of internal fixation was adopted. The inspected eye looks at the blinking gaze point in the lens. Taking the macula of the examined eye as the scanning site, taking the deepest concave of fovea as the center, six $6 \mathrm{~mm}$ radial scans with an intersection angle of 30 were performed, with 128 scanning points in one direction, totaling 768 points. The most concave part of the center concave should be near the 64th point. When deviating from this position, the fixation point should be adjusted to coincide with the intersection of scanning lines.

(3) Measurement of eye axis length

The eye axis length was measured by AI-2000 Measuring A Supermeter (Tomey Company, Japan). The measurement work was carried out by the same doctor with rich practical experience, and the measurement was repeated three times, and the average value was taken.

(4) Observation index of macular fovea thickness

Dividing around the macula, taking the fovea of macula as the center, selecting three concentric circles with diameters of $1 \mathrm{~mm}, 3 \mathrm{~mm}$ and $6 \mathrm{~mm}$ respectively, and adding two intersecting lines to divide the macula into inner ring area and outer ring area. The inner ring area refers to the area between circles with diameters of $1 \mathrm{~mm}$ and $3 \mathrm{~mm}$, also known as the inner macular area; While the 
circular area with a diameter of $1 \mathrm{~mm}$ in the inner ring area is the central concave area.

\subsection{Statistical analysis}

SPSS 19.0 software was used for statistical analysis. Application mean of quantitative data \pm The correlation between the equivalent spherical power and macular retinal thickness was analyzed by linear regression. $\mathrm{P}<$ 0.05 means the difference is statistically significant.

\section{$4 \quad$ Result}

\subsection{Measurement results of eye axis length}

See Table 1 for the comparison of gender, age, equivalent spherical lens and axial length between the two groups of children. There was no significant difference in age and sex between the two groups $(P>0.05)$. The length of equivalent spherical lens and eye axis in moderate and low myopia group was longer than that in emmetropia group, and the difference was statistically significant $(\mathrm{P}<$ $0.05)$.

Table 1. Basic data of two groups of patients

\begin{tabular}{llll}
\hline Project & Emmetropia group & $\begin{array}{l}\text { Moderate and low } \\
\text { myopia group }\end{array}$ & $\mathrm{P}$ \\
\hline Gender (male/female) & $28 / 22$ & $30 / 28$ & 0.14 \\
\hline Age (years) & $9.68 \pm 1.53$ & $9.30 \pm 1.34$ & 0.11 \\
\hline $\begin{array}{l}\text { Equivalent spherical } \\
\text { mirror (D) }\end{array}$ & $+0.25 \pm 0.23$ & $-2.50 \pm 1.33$ & $<0.01$ \\
\hline Eye axis length $(\mathrm{mm})$ & $23.52 \pm 0.56$ & $24.34 \pm 0.63$ & $<0.01$ \\
\hline
\end{tabular}

\subsection{Retinal thickness of macular area in middle and low myopia group and emmetropia group}

The macular retinal thickness of children in moderate and low myopia group and emmetropia group is shown in Table 2. There is no significant difference in
CMT(Central Macular Thickness) between the two groups ( $\mathrm{P}=0.514)$. MV(Macular Volume), AMT(Average Macular Thickness), I-AMT(average macular thickness of inner circle) and O-AMT(average macular thickness of outer circle) in moderate and low myopia group are lower than those in emmetropia group, and the differences are all statistically significant $(\mathrm{P}=0.000)$.

Table 2. Comparison of macular retinal thickness between moderate and low myopia and emmetropia group

\begin{tabular}{lllll}
\hline Project & $\mathrm{CMT}(\mu \mathrm{m})$ & $\mathrm{I}-\mathrm{AMT}(\mu \mathrm{m})$ & $\mathrm{O}-\mathrm{AMT}(\mu \mathrm{m})$ & $\mathrm{AMT}(\mu \mathrm{m})$ \\
\hline $\begin{array}{l}\text { Moderate and } \\
\text { low myopia } \\
\text { group }\end{array}$ & $223.78 \pm 18.24$ & $238.73 \pm 20.15$ & $245.72 \pm 17.25$ & $236.07 \pm 18.16$ \\
\hline $\begin{array}{l}\text { Emmetropia } \\
\text { group }\end{array}$ & $220.93 \pm 15.33$ & $236.73 \pm 18.14$ & $269.72 \pm 18.11$ & $242.46 \pm 18.21$ \\
\hline $\mathrm{P}$ value & $\mathrm{P}>0.05$ & $\mathrm{P}>0.05$ & $\mathrm{P}<0.05$ & $\mathrm{P}<0.05$ \\
\hline
\end{tabular}

\subsection{Correlation analysis of retinal thickness in different parts of macula in patients with moderate and low myopia}

In patients with moderate to low-grade myopia, the thickness of the retina below the macula and the nasal side was positively correlated with the refractive power $(\mathrm{r}=0.201,0.302, \mathrm{P}<0.05)$, and the retinal thickness of the whole macula was negatively correlated with the refrac- tive power $(\mathrm{r}=-0.326, \mathrm{P}<0.05)$; The thickness of the retina below the macula, the nasal side and the whole macula was negatively correlated with axial length $(\mathrm{r}=-0.220$, $0.314,-0.210, \mathrm{P}<0.05)$. There was no correlation between the thickness of the retina above the macula and the temporal side with refractive power and axial length $(\mathrm{r}=0.522,0.501,-1.121,-1.101, \mathrm{P}>0.05)$ (Table 3$)$.

Table 3. Correlation analysis of retinal thickness in different parts of macula in patients with moderate and low myopia

\begin{tabular}{|c|c|c|c|c|c|}
\hline Index & Diopter & Eye axis length & Index & Diopter & Eye axis length \\
\hline Above & & & $\mathrm{P}$ value & $<0.01$ & $<0.01$ \\
\hline r value & 0.522 & -1.121 & Temporal side & & \\
\hline $\mathrm{P}$ value & 0.081 & 0.325 & r value & 0.501 & -1.101 \\
\hline \multicolumn{2}{|c|}{ Lower position or level } & & $\mathrm{P}$ value & 0.063 & 0.214 \\
\hline r value & 0.201 & -0.220 & Full perimeter & & \\
\hline$P$ value & $<0.01$ & $<0.01$ & r value & -0.326 & -0.210 \\
\hline
\end{tabular}




\begin{tabular}{llllll}
\hline Nasal side & & & P value & $<0.01$ & $<0.01$ \\
\hline r value & 0.302 & -0.314 & & & \\
\hline
\end{tabular}

\section{Discussion}

No matter myopia or hyperopia, the retinal nerve fiber layer changes uniformly in different degrees. Among them, the thinning of retinal nerve fiber layer thickness in myopia is one of the most influential fundus changes in myopic retinopathy, which will not only produce corresponding complications, but also complicate the diagnosis of glaucoma in myopia. OCT can obtain the thickness of retinal nerve fiber layer objectively, accurately and non-invasively, which makes it possible to monitor and evaluate this index for a long time. OCT can scan the retina accurately and quickly without touching the human body, thus forming the corresponding OCT image. This scanning method not only has high resolution and strong security, but also can measure repeatedly until the satisfactory image is obtained.

The appearance of frequency-domain OCT provides a reliable method for measuring macular retinal thickness, which has twice the resolution and more than 60 times the scanning speed compared with time-domain OCT, and almost no motion artifacts. In this study, the high resolution, high sensitivity and ultra-fast acquisition speed of OCT in frequency domain are used, and most children can cooperate with it. There is a difference between this study and previous studies in macular retinal thickness, which is about $50 \mu \mathrm{m}$, mainly due to the different definition of retinal boundary by equipment. Our device defines retinal thickness as the distance from the inner limiting membrane to the inner surface of retinal pigment epithelium.

Some studies suggest that with the increase of myopia degree, the axial length of the eye is prolonged, the peripheral retina lacks large blood vessels and optic nerve fibers, and becomes thinner due to insufficient stress to resist pulling and stretching, while the thinning of the peripheral retina can offset the pulling force on the whole retina, so the change of macular central retina thickness is not obvious [12]. There are also studies that with the deepening of myopia, the eye axis is prolonged, and the microcirculation of the posterior pole is disordered, which leads to retinal choroidal dystrophy, the destruction of the outer segment structure of cone cells, the relative disorder of the arrangement of retinal pigment epithelial cells, and the reduction of the number of nucleated cells, especially the inner core layer [13]. However, the retina in the central macular area is mainly composed of cone cells, lacking the structure of other retina layers, so the thickness changes little. Previous studies [14] found that there was no obvious correlation between retinal thickness in the central macular area and myopia diopter, while other studies [15] found that retinal thickness in the central macular area increased with the deepening of myopia diopter. The results of this study show that in patients with moderate to low-grade myopia, there is a positive correlation between the thickness of the retina below the macula and the nasal side of the retina and the refractive power, and the thickness of the retina around the macula has a negative correlation with the refractive power; Negative correlation; the thickness of the retina above the macula and the temporal side has no correlation with refractive power and axial length. That is to say, as the refractive power of myopia increases, the retinal thickness in the central area of the macula increases, while the retinal thickness in the outer ring area of the macula becomes thinner.

The results of correlation research in this paper are basically consistent with the above research, but when comparing the thickness, it is found that there is no significant difference between the two groups of retina in most areas. The reason may be that the number of cases of high myopia in the included subjects is small, and the difference in refractive power between the two groups is insufficient, so that the difference has not yet been reflected. Compared with the other three directions, the retina on the nasal side of macula is the thickest area. Therefore, when the thinning ratio is the same, the absolute amount of thinning is more, which may be one of the reasons why the thinning of the retina on the nasal side was observed in the experiment.

\section{Conclusion}

To sum up, this study found that the retinal thickness in the central macular area of school-age children with moderate to low myopia is unchanged, which can maintain better central corrected vision. With the deepening of myopia, the retinal thickness outside the central macular area becomes thinner, which may affect the visual field outside the central vision and cause the visual quality to decrease.

\section{References}

1. Sso A, Mol A, Xlm B, et al. Retinal Thickness and Microvascular Changes in Children With Sickle Cell Disease Evaluated by Optical Coherence Tomography (OCT) and OCT Angiography[J]. American Journal of Ophthalmology, 2020, 209:88-98.

2. Wang Y, Ye J, Shen M, et al. Photoreceptor Degeneration is Correlated With the Deterioration of Macular Retinal Sensitivity in High Myopia[J]. Investigative ophthalmology \& visual science, 2019, 60(8):2800.

3. Ong S S, Linz M O, X Li, et al. Retinal Thickness and Microvascular Changes in Children with Sickle Cell Disease Evaluated by Optical Coherence Tomography and Optical Coherence Tomography Angiography[J]. American Journal of Ophthalmology, 2019, 209.

4. Peng-Fei Z, Yue-Hua Z, Jing Z, et al. Analysis of Macular and Retinal Nerve Fiber Layer Thickness in Children with Refractory Amblyopia after Femtosecond Laser-assisted Laser In situ Keratomileusis: A Retrospective Study[J]. Chinese medical journal, 2017, 130(18):2234-2240.

5. Niu Lili and Hu Junxi. Changes of macular retinal thickness and visual function in patients with rhegmatogenous retinal detachment after vitrectomy and silicone oil tam- 
ponade[J]. journal of xinxiang medical college, 2019, 036(007):657-661.

6. Goh J P, Koh V, Chan Y H, et al. Macular Ganglion Cell and Retinal Nerve Fiber Layer Thickness in Children With Refractive Errors-An Optical Coherence Tomography Study[J]. Journal of Glaucoma, 2017:619-625.

7. Kuddusi T, Murat K. Retinal and Choroidal Thickness in Patients with High Myopia without Maculopathy[J]. Pakistan Journal of Medical Sciences, 2017, 33(6):1438-1443.

8. Abe R, Sakurai M, Hoshi F, et al. Surgical outcome of vitrectomy for macular hole retinal detachment in high myopia with peeling of internal limiting membrane and silicone oil tamponade[J]. Folia Japonica De Ophthalmologica Clinica, 2017, 10(4):283-288.

9. Wei-Qin, Liu, Dan-Dan, et al. Topographic distribution features of the choroidal and retinal nerve fiber layer thickness in Wenzhou schoolaged children[J]. International Journal of Ophthalmology, 2020, v.13(03):106-113.

10. Lou Lei Fang, Hu Meijun. Application of optical coherence tomography in macular degeneration of high myopia [J]. International Journal of Ophthalmology, 2018, v.18(05):178-181.

11. Lin P W, Chang H W, Poon Y C. Retinal Thickness Asymmetry in Highly Myopic Eyes with Early Stage of Normal-Tension Glaucoma[J]. Journal of Ophthalmology, 2021, 2021(11):1-9.

12. Qiu-Jian, Zhu M Y, Wang P, et al. Analysis of macular microvasculature and thickness after ICL implantation in patients with myopia using optical coherence tomography[J]. International Journal of Ophthalmology, 2020, v.13(12):112-118.

13. Li V V, Smoliakova G P, Egorov V V, et al. Relevance of the Problem of Myopia in School-age Children with Signs of Undifferentiated Connective Tissue Dysplasia[J]. Ophthalmology in Russia, 2018, 15(2S):58-64.

14. Yung-Sung L, Lai-Chu S, Chang S H, et al. Macular Structures, Optical Components, and Visual Acuity in Preschool Children after Intravitreal Bevacizumab or Laser Treatment[J]. American Journal of Ophthalmology, 2018, 192:20-30.

15. Ye J A, Park Y Y, Park S H, et al. Long term change of the optic disc and OCT parameters during myopic shift in children with large cup to disc ratio[J]. PLoS ONE, 2020, 15(7):e0235621. 